\title{
Suitable photo-resists for two-photon polymerization using femtosecond fiber lasers
}

\author{
7 Q1 V.P. Rajamanickam ${ }^{\text {a,b }}$, L. Ferrara ${ }^{\text {a }}$, A. Toma ${ }^{a}$, R. Proietti Zaccaria ${ }^{a}$, G. Das ${ }^{\text {a }}$, E. Di Fabrizio ${ }^{\text {c,d }}$, C. Liberale ${ }^{\text {a,d,* }}$ \\ ${ }^{a}$ Nanostructures, Istituto Italiano di Tecnologia, via Morego 30, Genova I-16163, Italy \\ ${ }^{\mathrm{b}}$ Università degli Studi di Genova, Genova 16145, Italy \\ ${ }^{c}$ Lab. BIONEM, Dipartimento di Medicina Sperimentale e Clinica, Università degli Studi “Magna Graecia” di Catanzaro, viale Europa, I-88100 Catanzaro, Italy \\ ${ }^{\mathrm{d}}$ KAUST (King Abdullah University of Science and Technology), Thuwal 23955-6900, Saudi Arabia
}

\section{A R T I C L E I N F O}

\section{Article history:}

Received 27 October 2013

Received in revised form 22 April 2014

Available online $\mathrm{xxxx}$

\section{Keywords:}

Femtosecond fiber laser (fs fiber laser)

Bisphenol A ethoxylate diacrylate

(BPA-EDA)

Isopropyl thioxanthone (ITX)

7-Diethylamino-3-thenoylcoumarin (DETC)

$4,4^{\prime}$ Bis(diethylamino) benzophenone

(BDEB)

Two-photon polymerization (TPP)
Accepted 24 April 2014

\begin{abstract}
A B S T R A C T
We present suitable materials with good optical and mechanical properties, simple processing, efficient and optimized for two-photon polymerization (TPP) with femtosecond fiber lasers. We selected readily available acrylic monomer Bisphenol A ethoxylate diacrylate (BPA-EDA) with three different photoinitiators (PIs), isopropyl thioxanthone (ITX), 7-diethylamino-3-thenoylcoumarin (DETC), and 4,4' bis(diethylamino) benzophenone (BDEB), since their absorption spectra match well with the laser wavelength at $780 \mathrm{~nm}$. These PIs grant efficient radical generation, reactivity and high solubility in acrylic monomers. Finally, good optical and mechanical properties are demonstrated by the fabrication of different micro-structures.
\end{abstract}

(C) 2014 Published by Elsevier B.V.

\section{Introduction}

Micro/nano scale three-dimensional (3D) structures are showing their full potential in different fields like photonics, electronics, telecommunication and biomedicine being exploited in applications such as MEMS, information storage, plasmonics, metamaterials and tissue scaffolding [1-4].

For the fabrication of three-dimensional structures different approaches have been employed such as self-assembly [1,3], layer-by-layer stacking of planar patterns realized with consolidated lithographic techniques [2,4], ink-jet printing [5], robotic deposition [6], LIGA (X-ray-lithography, electro-forming and molding) [7], focused-ion-beam [8,9] and laser holographic lithography [10]. While these techniques allows for fabrication of 3D structures, they show a few limitations as in the complexity of the structures that can be created (e.g. self-assembly and laser holography are appropriate for structures with periodic patterns) or in the time required by many successive fabrication steps.

* Corresponding author at: Biological and Environmental Sciences and Engineering Division, King Abdullah University of Science and Technology (KAUST), Building 2, Level 4, Room \# 4335, Thuwal 2395-6900, Saudi Arabia. Tel.: +966 (0) 12808 2855.

E-mail address: carlo.liberale@kaust.edu.sa (C. Liberale).
Direct laser writing (DLW) based two-photon polymerization [11], being a true 3D maskless lithography, with reproducible even sub $100 \mathrm{~nm}$ resolution [12], has then recently gained increasing attention in areas such as optics, biology and electronics [13-20]. A large number of applications have been reported for DLW TPP, ranging from photonic crystals, to metamaterials, plasmonics, micromachines, microfluidics, micro-optics and 3D scaffolds [21, 22].

DLW-TPP technique consists into focusing a pulsed near infrared (NIR) laser beam into a photopolymer, made by a proper mixture of a photo-initiator and a monomer, through a high numerical aperture (NA) microscope objective, leading to multiphoton absorption only at the focal volume. The simultaneous absorption of two-photons creates radicals starting from the photo-initiator, triggering the monomer for cross-linking only in the focal volume, without affecting the outer resin. Thereby, moving the sample in $3 \mathrm{D}$ with respect to the beam or vice-verse allows the fabrication of the 3D micro-nano structures with, in principle, no limitations in the complexity of the shape geometry.

Most experimental setups for TPP lithography rely on modelocked Ti:sapphire lasers [14-20,25-37,39] with a repetition rate of $80 \mathrm{MHz}$ and $100 \mathrm{fs}$ pulse, that can tune the wavelength over a wide near-infrared (NIR) range, thus being compatible with most photo-initiators (PIs) used in UV photolithography. Unfortunately
62 63 64 65 66 67 
the cost for the Ti:sapphire system is relatively high, so there's an increasing request for materials that could adapt to the emerging technology of the less expensive femtosecond fiber lasers [23,24] that have the constraint of working at a fixed NIR wavelength (usually $780 \mathrm{~nm}$ ). Therefore, we standardized a new material which can efficiently undergo TPP using femtosecond fiber laser.

\section{Method and materials}

Our optical setup (Fig. 1a) is based on a mode-locked Er-doped fiber laser (FemtoFiber Pro NIR, Toptica) lasing at $780 \mathrm{~nm}$, with a repetition rate of $80 \mathrm{MHz}$ and a $100 \mathrm{fs}$ pulse width. An acoustic optical modulator (AOM) is used to regulate the laser power at the sample plane. The beam passes through a telescope where it is expanded to overfill the back focal plane of a microscope objective. The beam is then directed toward the microscope objective after passing through a dichroic mirror (DM), which reflects most of the NIR beam and transmits in the visible spectral range.

A piezoelectric stage with a travel range of $300 \mu \mathrm{m}$ in the $x, y$ and $z$ coordinates is interfaced with a computer and driven by a custom written LabVIEW software. This program transmits the data, that is a point by point structure, and controls the movement of the piezo stage. A $60 \times$ dry objective with a $0.7 \mathrm{NA}$ is used to focus the incoming beam into the pre-polymer. The objective is equipped with a correction collar that is used to minimize the spherical aberration. A LED light illumination helps to monitor in real time the beam alignment, focusing and fabrication process; the sample image is collected by a CCD camera. A short wave pass filter cuts the laser excitation on the camera.
To overcome the limitation set by the fixed NIR wavelength, we have tested different monomers and photoinitiators with an absorption spectrum peaked around $400 \mathrm{~nm}$ (Fig. 2), thus compatible with our setup. In particular we selected a acrylic monomer with the following characteristics: simple preparation by drop casting, soluble in common solvents and inert towards aggressive ones, high cross-linking to resist swelling, stable at high temperature, low shrinkage, good mechanical properties, high transparency in the visible when polymerized, high optical quality and rapid polymerization. Especially polyfunctional acrylate of Bisphenol A ethoxylate diacrylate [25] has shown high photosensitivity, high refractive index, low shrinkage, thermal stability and good adhesion in the polymeric state for the optical, surface and mechanical properties.

Among the photo-initiators, we selected isopropyl thioxanthone (ITX, Sigma-Aldrich), 7-diethylamino-3-thenoylcoumarin (DETC, Exciton, USA), and 4,4' bis(diethylamino)benzophenone (BDEB, Sigma), since their absorption spectra match well with the laser wavelength at $780 \mathrm{~nm}$. Moreover these photo-initiators grant high radical generation, reactivity and high solubility in acrylic monomers, also leading to an improvement in terms of resolution how has been recently shown $[26,27]$.

Directly available monomer Bisphenol A ethoxylate diacrylate (BPA-EDA, Sigma-Aldrich) and the photoinitiators ITX, DETC and BDEB were used without further purifications. Monomer and the different PIs were mixed in the weight percentage ratio (98.5:1.5 for ITX: 99.75:0.25 for DETC and BDEB) as described in [26]. The material was kept under $30^{\circ}$ with stirring for $1 \mathrm{~h}$ to complete the dissolution of the photoinitiator into the monomer. The refractive index of the not exposed material is 1.54 and the exposed

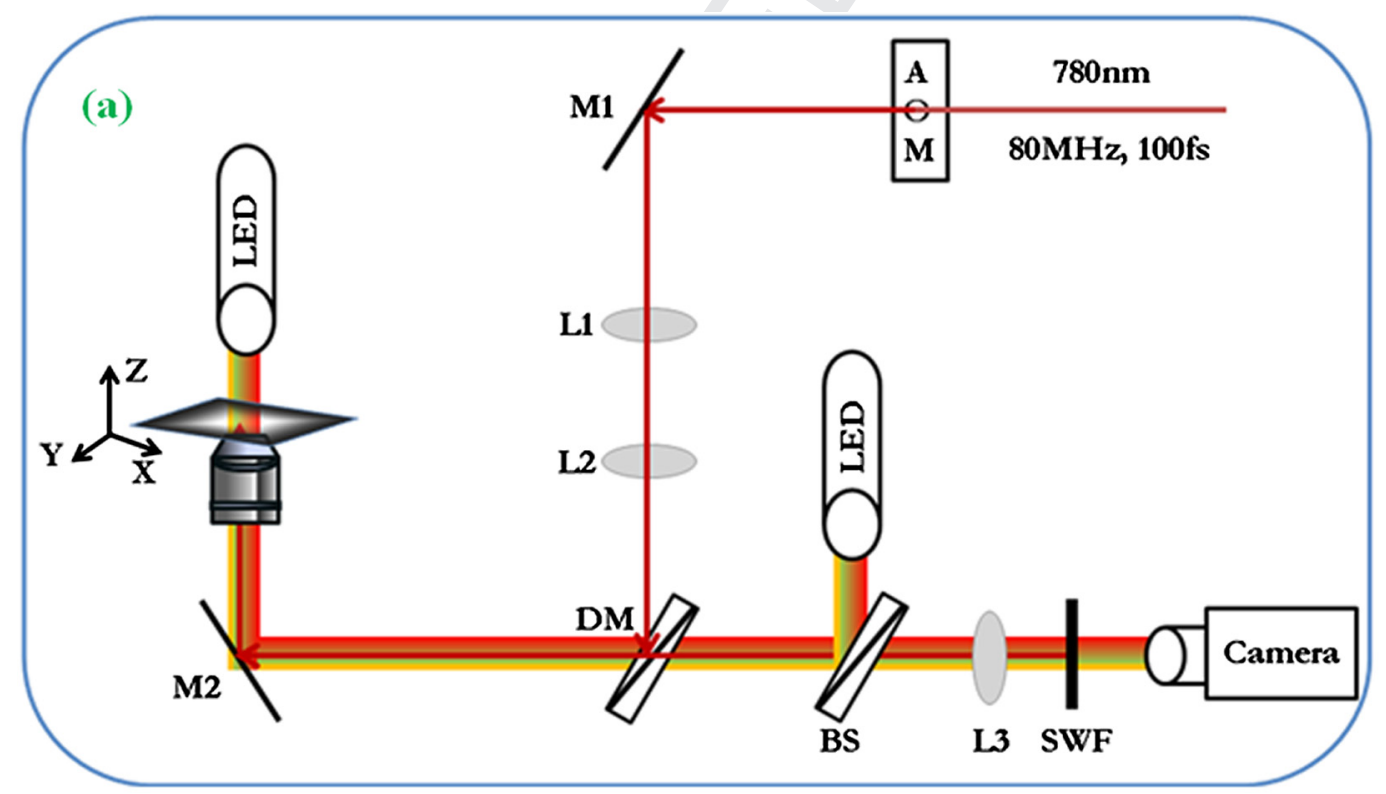

(b)

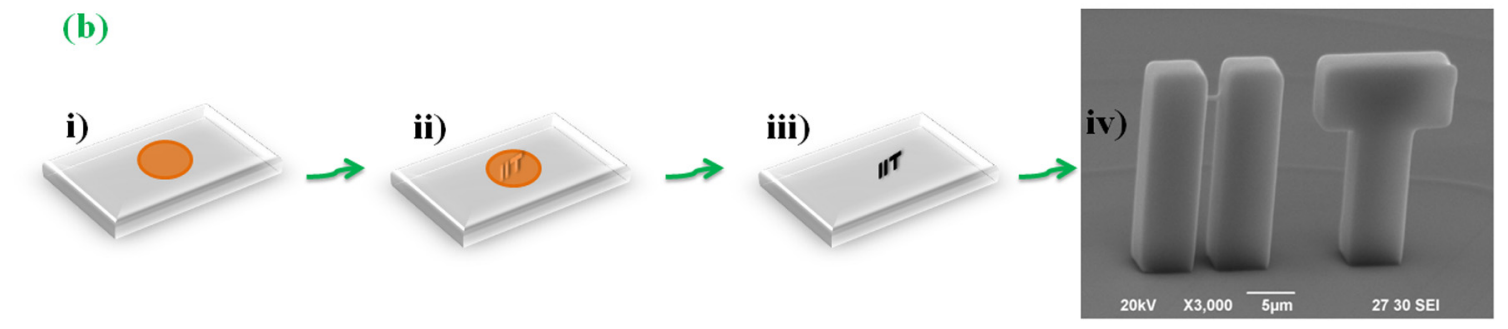

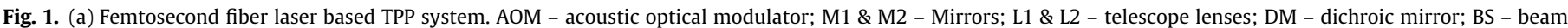

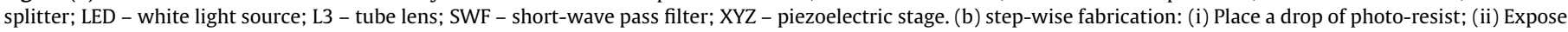
with TPP; (iii) Wash away the unexposed resist with solvents; (iv) SEM imaging and analysis. 


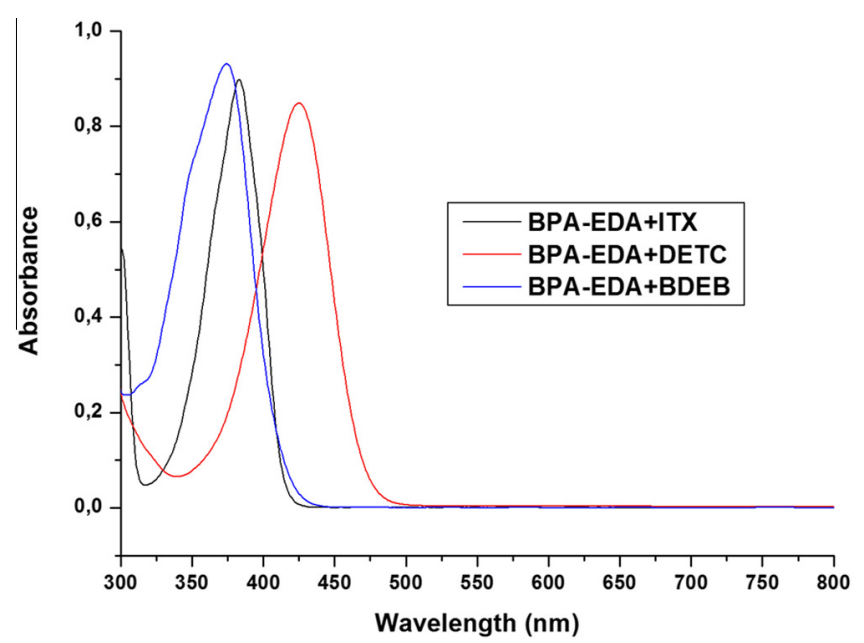

Fig. 2. Absorption spectra of monomer BPA-EDA in ITX, DETC and BDEB photoinitiators.

resists is around 1.59 [28]. A drop was then transferred into a cover slip (Fig. 1b) and placed on the xyz piezo with a holder.

Fabrication was performed following the scheme of single shell point by point exposure [29] that reduces fabrication time. Also, it allows having a self-smoothening [30] effect during the washing process of the unexposed part.

With this patterning strategy, structures such as asymmetric beads, micro-prisms, micro-spherical lenses and micro-cones were created. The exposure time for a single voxel was $10 \mathrm{~ms}$ and was optimized to have the shell thickness induced only by the voxels axial resolution and the lateral overlapping of $300 \mathrm{~nm}$. After the exposure, the material is developed with a rinse in methanol and isopropanol for a few seconds. The developed structures are then exposed with UV to complete the polymerization of the resin inside the inner volume.

We note that the whole process is very simple and it doesn't require additional pre- and/or post-processes as with other photocurable resists in literature [12,18-20,31-36] (e.g. SU 8, NOA63, Nopcocure 800 , hybrid sol-gel).

\section{Characterization}

The developed micro-structures were sputtered with $20 \mathrm{~nm}$ of gold for SEM analysis. The fabricated structures (Fig. 3a) prove the reliability of the proposed materials for fabrication of micro structures in terms of their size, geometrical shape, curvature, surface quality. They have also good adhesion property and low shrinkage after development.

In order to evaluate and compare the cross linking thresholds, we fabricated a micro-prisms structure with increasing power of the pulsed laser for the three different PIs (Fig. 3b). When exposed to the focused laser beam, the PI starts the polymerization with a minimum average-power threshold of around $1 \mathrm{~mW}$. If we increase the optical power by a few $\mathrm{mW}$, the polymerized structures achieve a good 3D mechanical stability, even with only the external surface exposed. We can see that BDEB has a higher cross linking threshold respect to ITX, but the latter needs a higher power to achieve enough mechanical stability with just a single-shell.

The achievable mechanical stability is further demonstrated with the fabrication of asymmetric bead structures, as seen from the Fig. 3a (top left), that find applications in optical tweezers (OTs)-based plasmonics [37]. This structure shows the potential of TPP to create arbitrary 3D geometries. For the optical trapping, the main body has three ellipsoidal bodies that act like handles, which are supported by a thin structure. The proposed material grants transparency and its good mechanical properties are evident from the thin structure which is able to withstand the relatively large top ellipsoid along with along protruding arm. For the plasmonic application, a metal nanocone, which acts as plasmonic probe, will be then fabricated by Electron Beam Induced Deposition [8] at the tip of the long arm.

Further, micro-optical components have been fabricated at the end facets of optical fibers. A miniaturized optical tweezers [38] based on optical fibers was recently proposed by exploiting beam deflection due to the total internal reflection (TIR) at the interface between fibers and the surrounding medium. At the point where the deflected beams converge together, there is the trapping point. To implement these deflecting structures by using TPP, we fabricated micro-prisms (Fig. 3a (top right)) with very smooth surface on the output facet of a fiber. On integrating this optical trapping function into a micro-fluidics chip, it has been demonstrated the trapping and spectroscopic analysis of single cells [20].

Similarly to the Ref. [39] we also demonstrate the fabrication of highly surface smoothened micro-lens Fig. 3a (bottom left)) with sizes on the order of tens to hundreds of micrometers for fiberbased imaging.

Additionally, high aspect ratio micro-cones (Fig. 3a (bottom right)) were fabricated for plasmonic terahertz applications [40].
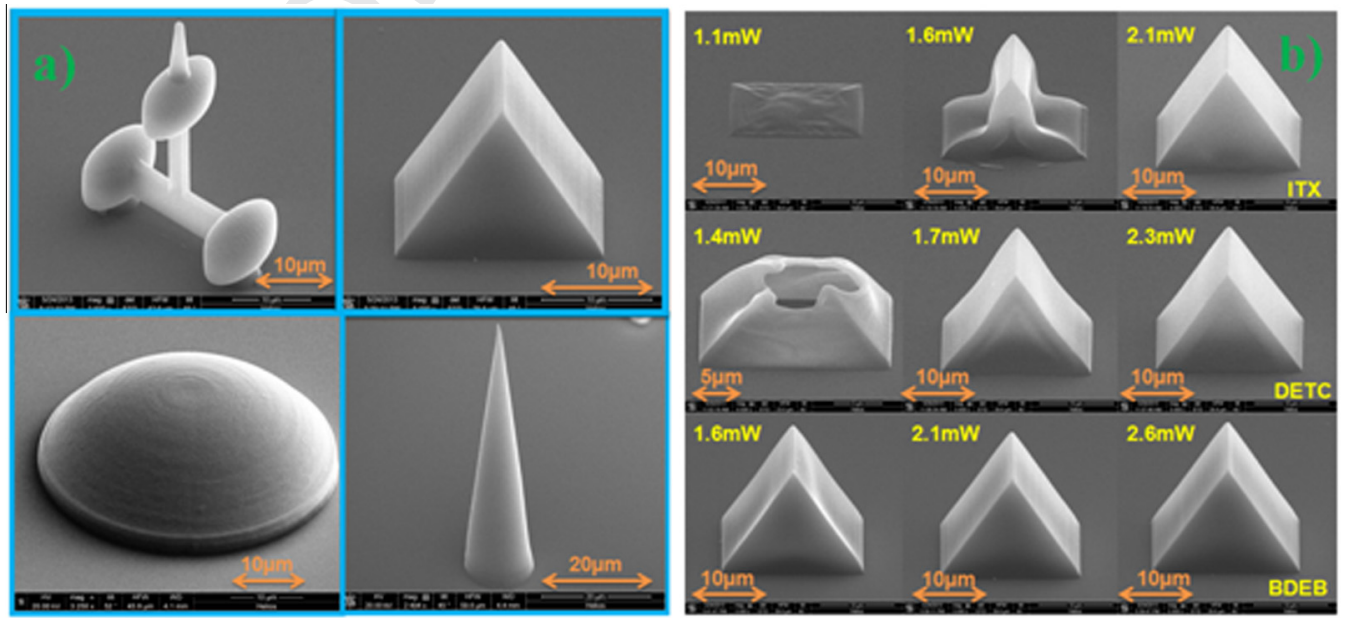

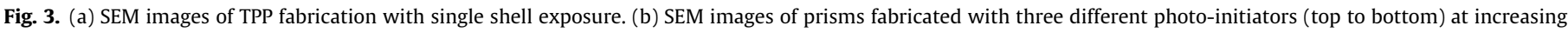
power (left to right). 

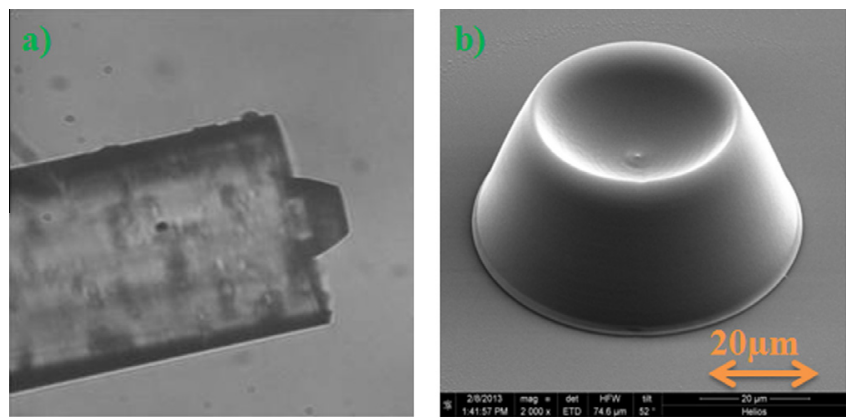

Fig. 4. (a) Optical image of a fabricated micro-parabolic reflector on top of multimode fiber. (b) SEM image of the micro-parabolic reflector.

At last, we have fabricated micro-parabolic reflectors (Fig. 4a and $b$ ) on top of multimode (MM) fibers to increase their numerical aperture (NA). This micro-fabricated optics [41] can be used in endoscopy through fiber imaging based on single MM fiber. The optical beam emerging from the fiber is reflected by the lateral parabolic surface due to the TIR. Fig. 4a shows the optical image of the micro-parabolic reflector as a beam deflector on a MM fiber. Their SEM images in the Fig. 4b clearly shows the smoothness of the lateral surfaces.

All these structures demonstrate the versatility of TPP with our proposed material for the fabrication of structures of both good optical and mechanical properties by using femtosecond fiber lasers.

\section{Conclusions}

We have proposed efficient and reliable materials for TPP using femtosecond fiber laser. The proposed PIs, due to their single photon absorption peaked around $400 \mathrm{~nm}$, generate radicals efficiently for TPP at the fixed wavelength of the femtosecond fiber laser. Optimized materials have simple processing with adequate optical and mechanical properties. SEM analysis shows satisfying properties of the fabricated structures in terms of geometrical shape, curvature, surface quality and mechanical stability. Glass matching refractive index, good adhesion to glass, and low shrinkage makes it promising for plasmonics and micro-optics.

\section{Acknowledgments}

We would like to thank Dr. Benjamin Harke for helpful discussions and for providing us the materials.

\section{References}

[1] G.M. Whitesides, J.P. Mathias, C.T. Seto, Science 254 (1991) 1312.

[2] R. Fabian Pease, S.Y. Chou, Proc. IEEE 96 (2008) 248.

[3] D. Qin, Y. Xia, G.M. Whitesides, Nat. Protoc. 5 (2010) 491-502.

[4] K.J. Stebe, E. Lewandowski, M. Ghosh, Science 325 (2009) 159.

[5] B.J. de Gans, P.C. Duineveld, U.S. Schubert, Adv. Mater. 16 (2004) 203-213.
[6] G.M. Gratson, M.J. Xu, J.A. Lewis, Microperiodic structures: direct writing of three-dimensional webs, Nature 428 (2004) 386

[7] J. Hruby, MRS Bull. 26 (2001) 337.

[8] F. De Angelis, G. Das, P. Candeloro, M. Patrini, M. Galli, A. Bek, M. Lazzarino, I. Maksymov, C. Liberale, L.C. Andreani, E. Di Fabrizio, Nat. Nanotechnol. 5 (2010) 67.

[9] D. Iannuzzi, K. Heeck, M. Slaman, S. de Man, J.H. Rector, H. Schreuders, J.W. Berenschot, V.J. Gadgil, R.G.P. Sanders, M.C. Elwenspoek, S. Deladi, Meas. Sci. Technol. 18 (2007) 3247-3252.

[10] S. Shoji, H.-B. Sun, S. Kawata, Appl. Phys. Lett. 83 (2003) 608.

[11] S. Maruo, O. Nakamura, S. Kawata, Opt. Lett. 22 (1997) 132-134.

[12] B.H. Cumpston, S.P. Ananthavel, S. Barlow, D.L. Dyer, J.E. Ehrlich, L.L. Erskine, A.A. Heikal, S.M. Kuebler, I.-Y.S. Lee, D. McCord-Maughon, J. Qin, H. RoÈckel, M. Rumi, X. Wu, S.R. Marder, J.W. Perry, Nature 398 (1999) 51.

[13] H.B. Sun, T. Kawakami, Y. Xu, J.Y. Ye, S. Matuso, H. Misawa, M. Miwa, R. Kaneko, Opt. Lett. 25 (2000) 1110.

[14] S. Kawata, H.-B. Sun, T. Tanaka, K. Takada, Nature (London) 412 (2001) $697-$ 698.

[15] T. Watanabe, M. Akiyama, K. Totani, S.M. Kuebler, F. Stellacci, W. Wenseleers, K. Braun, S.R. Marder, J.W. Perry, Adv. Funct. Mater. 12 (2002) 611-614.

[16] M. Deubel, G. Freymann, M. Wegener, S. Pereira, K. Busch, Nat. Mater. 3 (2004) 444-447.

[17] H.B. Sun, S. Kawata, in: Advances in Polymer Science: NMR, 3-D Analysis, Photopolymerization, Springer-Verlag, Berlin, 2004.

[18] C. Liberale, G. Cojoc, P. Candeloro, G. Das, F. Gentile, F. De Angelis, E. D Fabrizio, IEEE Photonics Technol. Lett. 22 (7) (2010) 474-476.

[19] M. Malinauskas, A. Zukauskas, K. Belazaras, K. Tikuisis, V. Purlys, R. Gadonas, A Piskarskas, Eur. Phys. J. Appl. Phys. 58 (2012) 20501.

[20] C. Liberale, G. Cojoc, F. Bragheri, P. Minzioni, G. Perozziello, R. La Rocca, L Ferrara, V. Rajamanickam, E. Di Fabrizio, I. Cristiani, Sci. Rep. 3 (2013) 1258.

[21] B.B. Xu, Y.L. Zhang, H. Xia, W.F. Dong, Lab. Chip 13 (2013) 1677-1690.

[22] Y.L. Zhang, Q.D. Chen, H. Xia, H.B. Sun, Nano Today 5 (2010) 435.

[23] W. Sibbett, A.A. Lagatsky, C.T.A. Brown, Opt. Express 20 (2012) 6989-7001.

[24] Dongchan Jang, Lucas R. Meza, Frank Greer, Julia R. Greer, Nat. Mater. 12 (2013) 893-898.

[25] S. Wu, M. Straub, M. Gu, Polymer 46 (2005) 10246-10255.

[26] B. Harke, W. Dallari, G. Grancini, D. Fazzi, F. Brandi, A. Petrozza, A. Diaspro, Adv. Mater. 25 (2013) 904-909.

[27] J. Fischer, M. Wegener, Laser \& Photon. Rev. 7 (2013) 22-44.

[28] S. Wu, G. Zhou, M. Gu, Opt. Mater. 29 (2007) 1793-1797.

[29] R. Guo, S.Z. Xiao, X.M. Zhai, J.W. Li, A.D. Xia, W.H. Huang, Opt. Express 14 (2006) 810 .

[30] D. Wu, S. Wu, L. Niu, Q. Chen, R. Wang, J. Song, H. Fang, Appl. Phys. Lett. 97 (2010) 031109.

[31] D. Palima, A.R. Bañas, G. Vizsnyiczai, L. Kelemen, P. Ormos, J. Glückstad, Opt Express 20 (2012) 2004-2014.

[32] M.R. Pollard, S.W. Botchway, B. Chichkov, E. Freeman, R.N.J. Halsall, D.W.K. Jenkins, I. Loader, A. Ovsianikov, A.W. Parker, R. Stevens, R. Turchetta, A.D. Ward, M. Towrie, New J. Phys. 12 (2010) 113056.

[33] D.B. Phillips, S.H. Simpson, J.A. Grieve, R. Bowman, G.M. Gibson, M.J. Padgett J.G. Rarity, S. Hanna, M.J. Miles, D.M. Carberry, EPL 99 (2012) 58004

[34] D. Palima, A.R. Bañas, G. Vizsnyiczai, L. Kelemen, T. Aabo, P. Ormos, J Glückstad, Opt. Express 21 (2013) 581-593.

[35] T. Asavei, T.A. Nieminen, V.L.Y. Loke, A.B. Stilgoe, R. Bowman, D. Preece, M.] Padgett, N.R. Heckenberg, H. Rubinsztein-Dunlop, New J. Phys. 15 (2013) 063016.

[36] B.L. Aekbote, J. Jacak, G.J. Schütz, E. Csányi, Z. Szegletes, P. Ormos, L. Kelemen, Eur. Polymer J. 48 (2012) 1745-1754.

[37] F. De Angelis, C. Liberale, M.L. Coluccio, G. Cojoc, E. Di Fabrizio, Nanoscale 3 (2011) 2689-2696.

[38] C. Liberale, P. Minzioni, F. Bragheri, F. De Angelis, E. Di Fabrizio, I. Cristiani, Nat Photonics 1 (2007) 723-727.

[39] G. Cojoc, C. Liberale, P. Candeloro, F. Gentile, G. Das, F. De Angelis, E. Di Fabrizio, Microelectron. Eng. 87 (2010) 876-879.

[40] L. Razzari, A. Toma, M. Shalaby, M. Clerici, R. Pi Zaccaria, C. Liberale, S. Marras, I.A. Al-Naib, G. Das, F. De Angelis, M. Peccianti, A. Falqui, T. Ozaki, R. Morandotti, Enzo Di Fabrizio, Opt. Express 19 (27) (2011) 26088-26094.

[41] S. Bianchi, V.P. Rajamanickam, L. Ferrara, E. Di Fabrizio, C. Liberale, R. Di Leonardo, Opt. Lett. 38 (23) (2013) 4935-4938. 